\title{
Minimally invasive management of white spot lesion using resin infiltration technique: A case report
}

\author{
(1) Ishani Saluja, (1) Sreelakshmi Pradeep, (1) Neeta Shetty
}

Department of Conservative Dentistry and Endodontics, Manipal College of Dental Sciences, Mangalore. Affiliated to Manipal Academy of Higher Education, Manipal, Karnataka, India

\section{Date submitted:}

28.12.2020

Date accepted:

11.02.2021

Online publication date: 15.03.2022

\section{Corresponding Author: \\ Neeta Shetty, MDS, Prof, Department of Conservative Dentistry and Endodontics, Manipal College of Dental Sciences, Mangalore. Affiliated to Manipal Academy of Higher Education, Manipal, Karnataka, India neeta.shetty@manipal.edu}

ORCID:

orcid.org/0000-0003-3693-5636

Keywords: Remineralization, resin infiltration, white spot lesion

\begin{abstract}
The resin infiltration technique is a non-invasive conservative method to manage initial carious lesions that appear as white spots on the enamel surface. The penetration of low viscosity resin into the enamel diminishes the white spot lesion immediately. The resin infiltration technique can be considered an alternative to micro-abrasion, macro-abrasion, and restorative treatment to treat the white spot lesions. This case report describes a technique used to arrest and mask the white spot lesions.
\end{abstract}

\section{Introduction}

Dental caries is considered one of the most prevalent chronic diseases worldwide (1). The first sign of dental caries is usually a white spot on the enamel surface when the tooth is dry. These post-eruptive white spots occur due to the demineralization of the enamel. Subsurface demineralization occurs beneath the intact enamel surface, creating an illusion of an opaque white spot. The appearance of the milky white lesion on the enamel surface is associated with the differences in the refraction of light on the lesion compared to sound enamel (2). Etiological factors for the white spots include early caries because of plaque accumulation around orthodontic brackets or poor oral hygiene, fluorosis, medication, molar incisal hypo-mineralization, and traumatic hypo-mineralization (3). These lesions can be detected using fiber-optic transillumination and dyes such as rhodamine and fluorescein (4).

In the oral cavity, the cycle of demineralization and remineralization of the enamel occurs. The demineralization process occurs when the $\mathrm{pH}$ falls below 5.5 (critical $\mathrm{pH}$ ) and leads to the formation of a white spot. The remineralization process can halt the progression of these white spot lesions. Remineralization depends on factors such as $\mathrm{pH}$ and mineral bioavailability. Several remineralizing agents have been used to treat the white spot lesions. Casein phosphopeptide-amorphous calcium phosphate, bioglass, xylitol, nanohydroxyapatite, sodium tri-metaphosphate, dicalcium phosphate dehydrate, and argon laser are widely used for the remineralization of such lesions. Ozone can also be used for remineralizing white 
spot lesions $(5,6)$. However, a new concept of resin infiltration technique has also been introduced to eliminate the white spot lesions to enhance aesthetics.

This case report explains the ultraconservative approach of treating white spots using resin material-ICON (Table 1). The technique relies on the infiltration concept and helps treat white spot lesions on proximal regions and smooth surfaces.

\section{Case Presentation}

A 33-year old female patient reported to the Department of Conservative Dentistry and Endodontics complaining of a white spot on the upper front tooth region for two months. She had undergone oral prophylaxis previously. The intraoral examination revealed a white spot lesion on the maxillary right canine (Figure 1a). The patient was informed about various treatment options, and the most conservative approach, resin infiltration technique, was chosen.

After obtaining the informed consent from the patient, oral prophylaxis was performed, followed by polishing using rubber cups (Enhance Finishing System, Dentsply Sirona, North Carolina) and prophylaxis paste (Prisma GlossTM, Dentsply Sirona, North Carolina). Isolation was achieved using a rubber dam (Dental Dam, Coltene, USA) (Figure 1b).

Icon-Etch $\mathrm{HCl}$ 15\% (Icon, Dental Milestones Guaranteed) was placed on the white spot lesion using an applicator tip for $2 \mathrm{~min}$. The tooth surface was rinsed for 30 seconds to remove the acid, followed by drying with oil- and water-free air. The lesion was desiccated using lcon-dry (99\% ethanol) for 30 seconds, which helped remove water from the pores of the lesion. On visual examination, the white spot was still visible, hence, Icon-Etch and Icon-Dry were reapplied. Whitish opaque lesion diminished significantly after the reapplication, following which Icon-resin was applied on the lesion for three minutes. The excess resin was removed from the facial surface using an applicator tip followed by interdental flossing $(7,8)$. It was then light-cured (3MTM ESPETM Dental: EiparTM 2500 Curing Light, Australia) for 40 seconds. Icon resin was reapplied on the surface for one minute. Excess material was removed and lightcured for 40 seconds. Polishing was carried out using rubber cups and polishing paste (Prisma GlossTM, Dentsply Sirona, North Carolina). The surface of the lesion was then covered with a layer of nanohybrid composite (Filtek Z 350, 3M ESPE, USA) and light-cured for 20 seconds. Polishing was carried out using Sof-Lex polishing discs (3M ESPE, USA) (Figure 1c).

\section{Discussion}

White spots occur due to subsurface demineralization of enamel beneath the hyper-mineralized superficial enamel surface (9). Because of differences in refractive indices of enamel, water, and air, white spot lesions appear more prominent when the tooth is dried. The refractive index of sound enamel is 1.62. When enamel is demineralized, it becomes more porous. When the tooth is desiccated, water from these porosities is replaced by air (refractive index 1.0) and becomes more opaque compared to the sound enamel. These white spots become less visible when these micro-porosities get filled with water (refractive index: 1.33). However, when these micro-porosities get filled with resin infiltrant, their refractive index becomes 1.52. Hence, the difference between the refractive indices of infiltrated lesion and enamel becomes negligible, and the lesions appear similar to the surrounding sound enamel (2).

Infiltration with resin helps mask the spot, even in the deeper regions of the lesion. The resin infiltration technique diminishes the white spots immediately compared to remineralizing agents. This technique is a more conservative approach, as it is less invasive than micro-abrasion, macro-abrasion, or restorations (9). Instead of removing the lesion, this technique helps to arrest incipient lesions by blocking the diffusion of acid into the enamel. Enamel also gets mechanically strengthened with the help of this resin infiltration.

Etching helps to erode the superficial hyper mineralized enamel layer and expose the body of the lesion. The hypermineralized enamel on the surface is removed by the etchant which helps the resin infiltrate to the ceiling of the lesion. According to Meyer-Lueckel et al. $(10,11), 15 \% \mathrm{HCl}$ is considered suitable for removing the hyper-mineralized surface layer (approximately

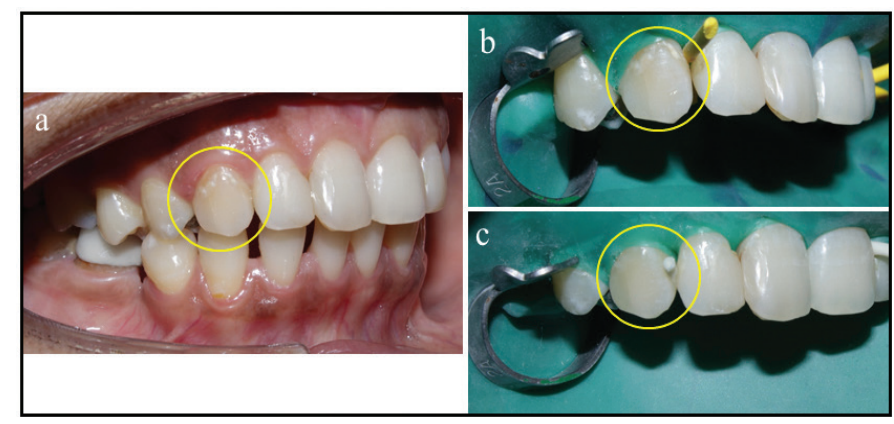

Figure 1. a) Initial situation. b) Preoperative picture (after rubber dam placement), c) Postoperative picture

\begin{tabular}{|lll|}
\hline \multicolumn{4}{|l}{ Table 1. Composition of resin infiltration kit (Icon) } & \\
\hline Commercial name (manufacturer) & Composition & Quantity \\
\hline Icon-etch (dental milestones guaranteed) & $15 \%$ hydrochloric acid, water, pyrogenic silica, surfactant, pigments & $1 \mathrm{syringe}(0.45 \mathrm{~mL})$ \\
\hline Icon-dry (dental milestones guaranteed) & $99 \%$ ethanol & $1 \mathrm{syringe}(0.45 \mathrm{~mL})$ \\
\hline Icon-infiltrant (dental milestones guaranteed) & TEGDMA-based resin, initiators and stabilizers & $1 \mathrm{syringe}(0.45 \mathrm{~mL})$ \\
\hline TEGDMA: Tetraethylene glycol dimethacrylate & & \\
\hline
\end{tabular}


$40 \mu \mathrm{m})$. Water present in the lesion's porosities is removed with the help of $99 \%$ ethanol, which permits the infiltrant to penetrate the pores driven by capillary forces (11).

Low viscosity, high surface tension, and low-contact angle enable the Icon Infiltrant to penetrate easily into the enamel (12). Compared with the other infiltrants, the Triethyleneglycol-dimethacrylate-resin infiltrant has shown deeper penetration. After applying for three minutes, tetraethylene glycol dimethacrylate-based infiltrant has shown to penetrate 414 microns into non-cavitated caries lesions. Resin is applied twice as there is a chance of shrinkage of the material after the first application, leading to the formation of spaces. The second application of resin helps to completely occlude these spaces (13). A thin layer of nanohybrid composite must be applied over the infiltrated tooth surface as the resin has a high staining potential $(14,15)$. The resin infiltration technique is especially advantageous in the management of aesthetics of mild white spot lesions present post-orthodontic treatment. It can be used as an adjunctive therapeutic measure in the management of early caries lesions.

\section{Conclusion}

Resin infiltration is a micro-invasive approach for aesthetic treatment of white spots since no healthy tooth structure is removed. The enamel's resistance is increased by hampering the demineralization process by sealing the micropores, and it is also a suitable method to arrest further caries. This technique is easy to perform and painless.

\section{Ethics}

Informed Consent: Consent form was filled out by all participants.

Peer-review: Externally peer-reviewed.

\section{Authorship Contributions}

Surgical and Medical Practices: I.S., Concept: I.S., S.P., Design: I.S., Data Collection or Processing: I.S., S.P., N.S., Analysis or Interpretation: I.S., S.P., Literature Search: I.S., N.S., Writing: I.S., N.S.

Conflict of Interest: No conflict of interest was declared by the authors.

Financial Disclosure: The authors declared that this study received no financial support.

\section{References}

1. Marcenes W, Kassebaum NJ, Bernabé E, et al. Global burden of oral conditions in 1990-2010: a systematic analysis. J Dent Res. 2013;92:592-597.
2. Richter $A E$, Arruda $A O$, Peters $M C$, Sohn W. Incidence of caries lesions among patients treated with comprehensive orthodontics. Am J Orthod Dentofacial Orthop. 2011;139:657664.

3. Marouane O, Chtioui F. Transillumination-aided infiltration: A diagnostic concept for treating enamel opacities. J Esthet Restor Dent. 2020;32:451-456.

4. Karawia IM, Mohamed OS. The Effect of Ozone Gas Using Different Remineralizing Materials on Non-Cavitated CariesLike Lesions in Permanent Teeth. Oral Health Dent Manag. 2017;16:1-5.

5. Alhamed M, Almalki F, Alselami A, Alotaibi T, Elkwatehy W. Effect of different remineralizing agents on the initial carious lesions - A comparative study. Saudi Dent J. 2020;32:390-395.

6. Attal JP, Atlan A, Denis M, Vennat E, Tirlet G. Whit spots on enamel: Treatment protocol by superficial or deep infiltration (part 2). Int Orthod. 2014;12:1-31.

7. Rocha Gomes Torres C, Borges AB, Torres LM, Gomes IS, de Oliveira RS. Effect of caries infiltration technique and fluoride therapy on the color masking of white spot lesions. J Dent. 2011;39:202-207.

8. Newbrun E, Brudevold F. Studies on the physical properties of fluorosed enamel I. Microradiographic studies. Arch Oral Biol. 1960;2:15-20.

9. Stahl J, Zandona AF. Rationale and protocol for the treatment of non-cavitated smooth surface carious lesions. Gen Dent. 2007;55:105-111.

10. Perdigão J. Resin infiltration of enamel white spot lesions: An ultramorphological analysis. J Esthet Restor Dent. 2020;32:317-324.

11. Meyer-Lueckel H, Paris S, Mueller J, Cölfen H, Kielbassa AM. Influence of the application time on the penetration of different dental adhesives and a fissure sealant into artificial subsurface lesions in bovine enamel. Dent Mater. 2006;22:22-28.

12. Paris S, Meyer-Lueckel H, Cölfen H, Kielbassa AM. Penetration coefficients of commercially available and experimental composites intended to infiltrate enamel carious lesions. Dent Mater. 2007;23:742-748.

13. Robinson C, Brookes SJ, Kirkham J, Wood SR, Shore RC. In vitro studies of the penetration of adhesive resins into artificial caries-like lesions. Caries Res. 2001;35:136-141.

14. Cohen-Carneiro F, Pascareli AM, Christino MR, Vale HF, Pontes DG. Color stability of carious incipient lesions located in enamel and treated with resin infiltration or remineralization. Int J Paediatr Dent. 2014;24:277-285.

15. Rey N, Benbachir N, Bortolotto T, Krejci I. Evaluation of the staining potential of a caries infiltrant in comparison to other products. Dent Mater J. 201430;33:86-91. 